\title{
BMJ Open Pneumoperitoneum preconditioning for the prevention of renal function after laparoscopic partial nephrectomy: protocol for a double-blind randomised controlled trial
}

To cite: Zhou C, Xu L, Xu Z, et al. Pneumoperitoneum preconditioning for the prevention of renal function after laparoscopic partial nephrectomy: protocol for a double-blind randomised controlled trial. BMJ Open 2020;10:e032002. doi:10.1136/ bmjopen-2019-032002

- Prepublication history and additional material for this paper are available online. To view these files, please visit the journal online (http://dx.doi org/10.1136/bmjopen-2019032002).

Received 29 May 2019 Revised 13 February 2020 Accepted 24 April 2020

\section{Check for updates}

(c) Author(s) (or their employer(s)) 2020. Re-use permitted under CC BY-NC. No commercial re-use. See rights and permissions. Published by BMJ.

${ }^{1}$ Department of Urology, Nanjing First Hospital, Nanjing Medical University, Nangjing, Jiangsu,

China

${ }^{2}$ Nuclear Medicine Center Nanjing First Hospital, Nanjing Medical University, Nangjing, Jiangsu, China

Correspondence to

Dr Ruipeng Jia;

urojiarp@njmu.edu.cn

\section{ABSTRACT}

Introduction Renal ischaemia reperfusion injury is an inevitable pathophysiology in different clinical situations including laparoscopic partial nephrectomy (LPN), which can obviously decrease the renal function after surgery. Pneumoperitoneum preconditioning (PP) is a promising approach that can yield a protective effect on kidney, which has already been demonstrated in some animal models. The present study is designed to assess whether the PP can yield a clinical renoprotective role after LPN. Methods and analysis This study is a randomised, prospective, double-blind and parallel controlled clinical trial. Eligible participants will be patients with renal tumours and willing to choose elective LPN. Patients randomised to the treatment arm will receive PP consisted of three cycles of 5 min insufflation and 5 min desufflation before LPN, while the control arm will receive a sham operation. The primary endpoints are glomerular filtration rate and the level of serum cystatin $C$ within 6 months after desufflation. The secondary endpoints are serum creatinine, estimated glomerular filtration rate, alanine transaminase, serum amylase, intestinal fatty acid binding protein, postoperative hospital stay, the incidence of adverse events and mortality in postoperative 6 months. Ethics and dissemination This study has been approved by the institutional ethics committee of Nanjing First Hospital. The results of this study will be reported faithfully through scientific conferences or published articles. Trial registration number NCT03822338.

\section{INTRODUCTION}

Partial nephrectomy (PN) has become the standard surgical option for small renal tumours, which can preserve the maximum functional renal tissues on the basis of complete tumour removal. ${ }^{12}$ Compared with traditional open operation, laparoscopic PN (LPN) can be more acceptable for the merits of minimal invasion and rapid recovery. ${ }^{3}$ In most cases, the renal artery needs to be occluded during LPN to facilitate the surgery, which can result in renal ischaemia

\section{Strengths and limitations of this study}

- This will be the first double-blind randomised controlled trial to investigate the efficacy and safety of pneumoperitoneum preconditioning in patients with renal tumours after laparoscopic partial nephrectomy.

${ }^{99 \mathrm{~m}} \mathrm{Tc}$-diethylenetriaminepentaacetic acid $\left({ }^{99 \mathrm{~m}} \mathrm{TC}\right.$-DTPA) renal scintigraphy will be used to detect the change in glomerular filtration rate of unilateral impaired kidney.

- Standard Protocol Items Recommendations for Interventional Trials checklist guidelines were followed when we drafted this protocol.

- Limitations may be the relatively small sample size, short follow-up time, and not fully controlled warm ischaemia time during surgery.

reperfusion injury (IRI) and affect the postoperational renal function. ${ }^{45}$ Moreover, the increase of intra-abdominal pressure in LPN can decrease the blood flow of abdominal viscera including kidney, and further aggravate the renal IRI. ${ }^{6}$ Several strategies have been proposed to reduce renal IRI during LPN. $^{7-9}$ However, most methods are relatively complicated to implement or merely limited to surgical expertise. Therefore, an effective and feasible strategy is urgently warranted to prevent renal function after LPN.

Ischaemic preconditioning (IP) is a novel non-pharmacological approach, during which the target organ is conditioned with a brief episode of ischaemia and reperfusion prior to the subsequent prolonged ischaemic insult, and it has been confirmed effective in the alleviation of renal IRI. ${ }^{10}$ However, the preoperative administration of IP through clamping the renal artery would be impractical to apply. Pneumoperitoneum preconditioning (PP) is 


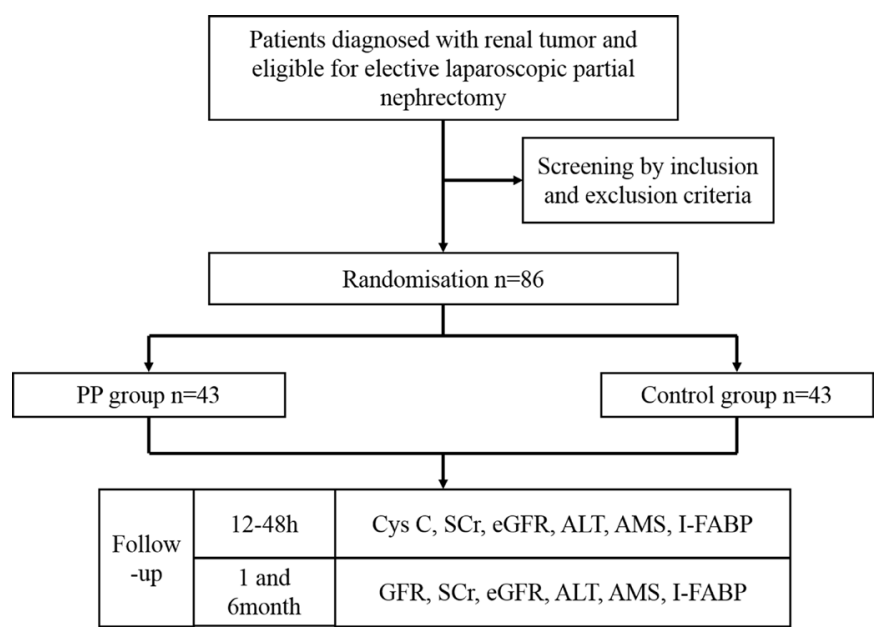

Figure 1 Schematic flow chart of the study. ALT, alanine transaminase; AMS, amylase; Cys C, cystatin C; eGFR, estimated glomerular filtration rate; GFR, glomerular filtration rate; I-FABP, intestinal fatty acid binding protein; PP, pneumoperitoneum preconditioning; $\mathrm{SCr}$, serum creatinine.

one type of IP, and the transient periods of ischaemia and reperfusion during $\mathrm{PP}$ are executed by mechanical insufflation and desufflation, which can be relatively more safe and feasible than artery clamping. ${ }^{611} 12$ Numerous animal researches have been conducted to detect the protective role of PP on organ IRI and explore the potential mechanisms. ${ }^{13}{ }^{14}$ Unfortunately, the utilisation of PP is rarely practised in clinical setting.

Therefore, we designed a randomised controlled trial (RCT) to assess the short-term and long-term renoprotective role of PP in patients undergoing LPN, and observe its effect on the other abdominal organs.

\section{METHODS AND ANALYSIS}

\section{Study design}

The present study will be a randomised, prospective, double-blind and parallel controlled clinical trial based on a single centre, and has already been successfully registered at ClinicalTrials.gov. The included participants in this study will be patients with renal tumours undergoing elective LPN between 2019 and 2020 in Nanjing First Hospital, Nanjing Medical University. Eligible patients will be randomly assigned to control group or PP group with a 1:1 ratio, and the follow-up will last for 6 months. A brief flow diagram of our study protocol is presented in figure 1. This study protocol has been designed and written in accordance with the Standard Protocol Items Recommendations for Interventional Trials 2013 Checklist. ${ }^{15}$

\section{Protocol version}

Issue date: 9 January 2019; version identifier: NFH20180205; number of protocol amendments: 5; initial version: 16 February 2018. First modification: 3 March 2018, the procedure of PP was altered to three cycles of 5 min insufflation and 5 min desufflation. Second modification: 10 May 2018, the inclusion criteria regarding renal tumour was changed to stage Tla and tumour $\leq 4 \mathrm{~cm}$ in diameter, and the corresponding exclusion criteria was also reset (tumour $>4 \mathrm{~cm}$ in diameter). Third modification: 5 August 2018, alanine transaminase (ALT), serum amylase (AMS) and intestinal fatty acid binding protein (I-FABP) were added as secondary endpoints. Fourth modification: 22 November 2018, the estimation of sample size was optimised. Fifth modification: 9 January 2019, optimising of inserted figures and formula.

\section{Eligibility criteria \\ Inclusion criteria}

(1) Male and female patients aged 18 years and older; (2) patients with benign or malignant renal tumours who choose LPN as the surgical method; (3) the renal tumour must be stage Tla and no more than $4 \mathrm{~cm}$ in diameter (assessed by MRI/CT); (4) the unilateral renal tumour, and another contralateral renal function reveal normal (renal function $>40 \%$ as determined by radionuclide scintigraphy); (5) patients volunteer for this study and provided written informed consent.

\section{Exclusion criteria}

(1) Large tumours (tumours $>4 \mathrm{~cm}$ in diameter); (2) severe renal insufficiency (glomerular filtration rate $(\mathrm{GFR})<30 \mathrm{~mL} / \mathrm{min} / 1.73 \mathrm{~m}^{2}$ ) or needing preoperative haemodialysis or peritoneal dialysis; (3) patients with severe cardiopulmonary dysfunction; (4) pregnant women; (5) patients with other malignant tumours; (6) patients with a history of other abdominal viscera operations within 1 year (open or under laparoscope); (7) American Society of Anesthesiologists Physical Status Classification System for patients (ASA)>III; (8) patients who have had a renal transplantation.

\section{Recruitment, randomisation and allocation}

All the participating researchers will be systematically trained before the beginning of the trial. The screening of the patients will be conducted by two experienced urologists based on the above criteria. Discrepancies between the two researchers will be resolved via discussion with the other senior surgeon. A well-trained researcher will describe and explain the detailed procedure of the study to the patients and answer any questions from them. Patients with written, voluntary, informed consent will be regarded as eligible for the following treatment assignment. An example of the patient consent form is presented as an online supplementary material.

Eligible patients will be randomly divided into two groups: the control group and the PP group according to a 1:1 ratio using a blocked randomisation with randomly varying block size ( 4 or 6 ). Participants will be grouped into randomisation blocks by the time and date of surgery. The randomisation will be accomplished using a computer-generated random sequence. The random assignment of each participant will be carried out by two independent researchers, and the serial number will be sealed in an opaque identical envelope, which must not be unfolded until the start of PP. The operator of the 


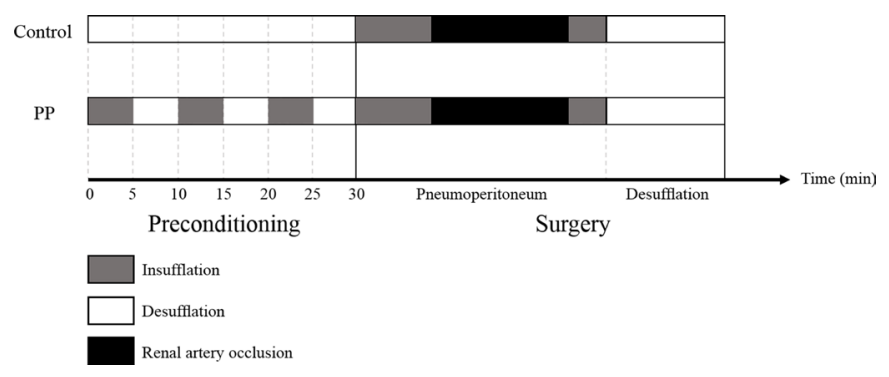

Figure 2 Schematic interpretation of the experimental protocol. PP, pneumoperitoneum preconditioning.

pneumoperitoneum machine will open the envelope and deliver the assigned treatment. To avoid potential bias, the surgeons, patients and outcome assessors will be blind to the group allocation.

\section{Intervention}

PP group

The detailed experimental protocol is presented in figure 2. Participants assigned to the PP group will receive a treatment consisting of three cycles of 5 min insufflation and 5 min desufflation, after complete anaesthesia and successfully implanting the veress. The pneumoperitoneum machine will be introduced to induce this process, and carbon dioxide gas will be used to maintain the intraabdominal pressure at $15 \mathrm{~mm} \mathrm{Hg}$. The whole duration will last $30 \mathrm{~min}$. The subsequent LPN technique has been described in detail previously, ${ }^{16}$ and the renal artery will be only clamped rather than the whole pedicle in our surgery.

\section{Control group}

Participants in the control group will receive the same placement of the veress but without insufflation and subsequent desufflation, this process will also similarly last $30 \mathrm{~min}$.

Use of nephrotoxic drugs or other treatments that can lead to renal injury is prohibited during the trial, due to their potential influence on the final results.

\section{Outcome measures}

\section{Primary endpoints}

(1) The change in unilateral (affected kidney and contralateral kidney) and overall GFR detected by ${ }^{99 \mathrm{~m}} \mathrm{Tc}$ diethylenetriaminepentaacetic acid ( ${ }^{99 m}$ Tc-DTPA) renal scintigraphy from baseline to postoperative 1 month and 6 months; (2) change in the level of serum cystatin C (Cys C) from baseline to 12 hours, 24 hours and 48 hours after desufflation.

\section{Secondary endpoints}

(1) The change in serum creatinine (SCr) from baseline to 12 hours, 24 hours, 48 hours, 1 month and 6 months after desufflation; (2) change in postoperative estimated glomerular filtration rate (eGFR) from baseline to 12 hours, 24 hours, 48 hours, 1 month and 6 months after desufflation; (3) the change in serum markers for detecting insults of other abdominal organs including
ALT, AMS and I-FABP (time frame: from baseline to 12 hours, 24 hours, 48 hours, 1 month and 6 months after desufflation); (4) the duration of postoperative hospital stay; (5) the incidence of adverse events (AEs) within 6 months; (6) mortality within 6 months. eGFR will be calculated by a combined formula through serum creatinine: $\mathrm{eGFR}=186 \times(\mathrm{SCr})^{-1.154} \times(\text { age })^{-0.203} \times 0.742 \quad$ (female) $\times 1.210$ (black).

Additionally, we will also record the following: (1) baseline characteristics of each patients including: age, gender, weight, pre-existing diseases and ASA Score; (2) the tumour characteristics: location, diameter, stage and Preoperative Aspects and Dimensions Used for an Anatomical (PADUA) Score; (3) perioperative data including: anaesthetic drugs (induction and maintenance), the duration of prolonged pneumoperitoneum, warm ischaemia time (WIT) and blood loss; (4) the preoperative baseline levels of GFR, Cys C, SCr, eGFR, ALT, AMS and I-FABP after admission.

The outcomes of all participants at baseline and 12 hours, 24 hours, 48 hours after desufflation will be recorded during the hospital stay. At 1 month and 6 months after LPN, patients will be seen at clinic visits for serum examination and GFR detecting. Telephone follow-up will also be performed monthly to monitor their status including AEs.

\section{Data collection and management}

All data will be recorded using a unified report form and collected and maintained in a confidential manner (identifying information of patients will be invisible and each participant will only be labelled by a random code throughout the experiment). All research data will be stored on a fixed server and backed up to another confidential server, with restricted access by primary researchers. Data will be stored for 5 years before being destroyed.

\section{Sample size calculation}

One of the primary objectives of the presently designed study will be the change in GFR of the affected kidney from baseline. Accordingly, we used the following formula according to the Chow et al's textbook ${ }^{17}$ presuming the change in primary endpoint (GFR) to estimate the required sample size per group:

$$
\begin{gathered}
n_{A}=\kappa n_{B} ; n_{B}=\left(1+\frac{1}{\kappa}\right)\left(\sigma \frac{z_{1-\alpha / 2}+z_{1-\beta}}{\mu_{A}-\mu_{B}}\right)^{2} ; \\
1-\beta=\Phi\left(z-z_{1-\alpha / 2}\right)+\Phi\left(-z-z_{1-\alpha / 2}\right) ; z=\frac{\mu_{A}-\mu_{B}}{\sigma \sqrt{\frac{1}{n_{A}}+\frac{1}{n_{B}}}}
\end{gathered}
$$

$\left(\kappa=n_{A} / n_{B}\right.$ is the matching ratio; $\sigma$ is $\mathrm{SD} ; \Phi$ is the standard normal distribution function; $\alpha$ is type I error; $\beta$ is type II error; $\mu_{A}-\mu_{B}$ is the mean difference between two groups).

We expect the between-group difference in the absolute change in GFR of the affected kidney from baseline to postoperative 6 months, according to a comprehensive reference to a previous study, ${ }^{18}$ will be $3.5 \mathrm{~mL} /$ $\min / 1.73 \mathrm{~m}^{2}$, with a SD of 4.75 . We assume a $10 \%$ rate of 
loss to follow-up. Given these assumptions, the t-test will require 43 patients per group to reach $90 \%$ power at twosided $\alpha$-level of $5 \%$.

\section{Statistical methods}

For continuous data which are consistent with the normal distribution, the outcomes will be expressed as mean $\pm \mathrm{SD}$. Otherwise, the median and IQR will be used. The repeated-measures analysis of variance will be used to determine the differences between the PP and control group over time. Results of Greenhouse-Geisser correction will be reported whenever Mauchly's test of sphericity is significant. The differences between the two groups at each time point will be further evaluated by analysis of covariance with baseline value for each endpoint as a covariate. For binary variables, the $\chi^{2}$ test or Fisher's exact will be introduced to explore the potential differences between groups. The kappa coefficient will be introduced to assess the consistency of the selection and data extraction. Imputations methods will be applied to account for missing data. Subgroup analysis with a test of interaction will be conducted to examine heterogeneity of treatment effect. The subgroup variables will include age, gender, PADUA Score and WIT. Results will be considered to be statistically significant at $p<0.05$. All data analysis will be performed by separate statisticians from the First Hospital of Nanjing using SPSS software V.24.0, and all of them will be blind to the randomisation and allocation.

\section{Monitoring}

An independent data monitoring committee (DMC) consisting of expert clinicians, statisticians and ethicists will monitor the efficacy and safety of interventions, audit the integrity and overall quality of the data and check the compliance with the protocol. Visits will be monitored monthly during the trial. The DMC is independent of the sponsor and has no competing interests. There will be no interim analysis as the study procedures and data will be regularly checked by the DMC every month. The DMC will discuss the results with the ethics committee and steering committee to decide whether the trial can continue. If there is an unacceptable risk of serious AEs, the study will be ceased.

The monitoring of $\mathrm{AE}$ will begin after the start of the detection of preoperative GFR baseline levels, and last 6 months after surgery. The relationship between interventions and $\mathrm{AE}$ will be determined by clinical specialist, and all $\mathrm{AE}$ will be recorded and included in the trial. Serious $\mathrm{AE}$ will be reported to the DMC and ethics committee within 24 hours.

\section{Patient and public involvement}

Patients and the public will not be involved in the process of this study including design, recruitment and conduction. All participants will be informed by a summary of the study results at the end of the trial.
Ethics and dissemination

This study has been approved by the institutional ethics committee of Nanjing First Hospital. Researchers need to explain the detailed experimental process to all participants, and written informed consents must be formally obtained from all patients before randomisation. All participants can freely withdraw from this experiment at any time with no reason. All related research data will be stored securely and not be released externally without the permission of patients.

Regardless of magnitude and trend of the final results, we will report it faithfully through scientific conferences or published articles.

\section{Ancillary and post trial care}

If patients suffer study-related harm after the trial, free medical care and adequate economic compensation will be provided.

\section{DISCUSSION}

Renal IRI is inevitable during LPN in most cases and is closely related to the decreased renal function after surgery. ${ }^{4-6}$ This signifies the urgency of more protective and practicable approaches. PP is a novel nonpharmacological strategy to induce IP, which can be easily and securely implemented before LPN through mechanical insufflation and desufflation. ${ }^{11} 12$ Encouragingly, PP has been proved to be an effective method for renoprotection against IRI in several animal models, ${ }^{13} 14$ and could yield positive effect of reducing oxidative stress after LPN. ${ }^{11}$ To the best of our knowledge, this trial will be the first clinical attempt to investigate the efficacy of PP in patients with renal tumours after LPN. Participants will be followed for 6 months to detect the short-term and long-term effect of PP.

The performed protocol of PP in our recent study will consist of three cycles of $5 \mathrm{~min}$ insufflation and $5 \mathrm{~min}$ desufflation instead of one cycle of 15 min insufflation and $15 \mathrm{~min}$ desufflation as done in previous studies. ${ }^{6}$ The prolonged insufflation with $15 \mathrm{~min}$ could cause a lethal injury to abdominal organs before LPN, although the total ischaemia and reperfusion duration of the two procedures are both $30 \mathrm{~min}$. To evaluate the short-term effect of PP on renal function, the postoperative level of Cys $\mathrm{C}$ is introduced as one of the primary outcomes, for Cys C is more sensitive than traditional SCr or blood urea nitrogen in the early detection of kidney injury. ${ }^{10} \mathrm{We}$ collect GFR detected by ${ }^{99 m}$ Tc-DTPA renal scintigraphy as the primary outcome to assess the unilateral and overall renal function after surgery, because it should be more accurate and specific than eGFR. Additionally, blood flow to the other abdominal viscera will also be decreased during surgical pneumoperitoneum inducing subsequent organ IRI. ${ }^{6} 1920$ The practical effect of additional pneumoperitoneum on the other abdominal viscera has not been clearly determined. A preclinical study in a porcine model conducted by Avraamidou et $a l^{21}$ demonstrated 
that PP can attenuate damage to the liver and kidney after intra-abdominal hypertension. In the present study, we will observe the levels of ALT, AMS and I-FABP to evaluate the postoperative degree of short-term and longterm insults of the liver, pancreas and intestine, which are more sensitive to IRI. ${ }^{12}$

There are several limitations of this study design that should be acknowledged. First, our trial is a single-centre study, which may not be generalisable to other centres. Second, the function of kidney and other organs will only be evaluated by outcomes measured within 6 months of treatment. Finally, WIT cannot be unified in common with standardised animal experiments, for it is closely associated with the characteristics of the tumours and the complexity of surgery.

In summary, this will be the first RCT to detect the renoprotective role of $\mathrm{PP}$ in patients undergoing LPN and assist with the determination of effective strategies for the prevention of IRI. The results yielded from this study will be helpful to improve the overall efficacy of LPN for patients with renal tumours.

Acknowledgements The authors wish to thank Jinkou Zhao for his generous assistance with this study.

Contributors RJ conceived this study idea. CZ, YG and LZ designed the study. JL and TY assisted in the design and refinement of the study protocol. LX, ZX, and RJ contributed for the refinement of the protocol with clinical expertise. CZ, FW and GP advised on the statistical plan and data analysis. CZ and YG drafted the manuscript, and all author contributed substantially to the edit of the manuscript. All authors approved the final version.

Funding This study was supported by Jiangsu Provincial Social Development Project (BE2017615 to RJ), and Postgraduate Research \& Practice Innovation Program of Jiangsu Province (JX22013530 to CZ). The funders had no role in study design, data collection and analysis, decision to publish or preparation of the manuscript.

\section{Competing interests None declared.}

Patient and public involvement Patients and/or the public were not involved in the design, or conduct, or reporting, or dissemination plans of this research.

\section{Patient consent for publication Not required.}

Provenance and peer review Not commissioned; externally peer reviewed.

Open access This is an open access article distributed in accordance with the Creative Commons Attribution Non Commercial (CC BY-NC 4.0) license, which permits others to distribute, remix, adapt, build upon this work noncommercially, and license their derivative works on different terms, provided the original work is properly cited, appropriate credit is given, any changes made indicated, and the use is non-commercial. See: http://creativecommons.org/ licenses/by-nc/4.0/.

\section{ORCID iD}

Ruipeng Jia http://orcid.org/0000-0002-5532-5641

\section{REFERENCES}

1 Scosyrev E, Messing EM, Sylvester R, et al. Renal function after nephron-sparing surgery versus radical nephrectomy: results from EORTC randomized trial 30904. Eur Urol 2014;65:372-7.

2 Huang WC, Levey AS, Serio AM, et al. Chronic kidney disease after nephrectomy in patients with renal cortical tumours: a retrospective cohort study. Lancet Oncol 2006;7:735-40.

$3 \mathrm{Ng} \mathrm{AM}$, Shah PH, Kavoussi LR. Laparoscopic partial nephrectomy: a narrative review and comparison with open and robotic partial nephrectomy. J Endourol 2017;31:976-84.

4 Godoy G, Ramanathan V, Kanofsky JA, et al. Effect of warm ischemia time during laparoscopic partial nephrectomy on early postoperative glomerular filtration rate. J Urol 2009;181:2438-45.

5 Funahashi Y, Hattori R, Yamamoto T, et al. Ischemic renal damage after nephron-sparing surgery in patients with normal contralateral kidney. Eur Urol 2009;55:209-16.

6 Leventi A, Argyra E, Avraamidou A, et al. Attenuation of oxidative stress by ischemic preconditioning in an experimental model of intraabdominal hypertension. J Invest Surg 2015;28:253-60.

7 Shao P, Qin C, Yin C, et al. Laparoscopic partial nephrectomy with segmental renal artery clamping: technique and clinical outcomes. Eur Urol 2011;59:849-55.

8 Gill IS, Patil MB, Abreu ALdeC, et al. Zero ischemia anatomical partial nephrectomy: a novel approach. J Urol 2012;187:807-15.

9 Naya Y, Kawauchi A, Yoneda K, et al. A comparison of cooling methods for laparoscopic partial nephrectomy. Urology 2008;72:687-9.

$10 \mathrm{Ge} \mathrm{Y-Z,} \mathrm{Wu} \mathrm{R,} \mathrm{Xin} \mathrm{H,} \mathrm{et} \mathrm{al.} \mathrm{Effects} \mathrm{of} \mathrm{ischemic} \mathrm{preconditioning} \mathrm{on}$ the systemic and renal hemodynamic changes in renal ischemia reperfusion injury. Int J Clin Exp Pathol 2015;8:1128-40.

11 Karabayirli $\mathrm{S}$, Surgit $\mathrm{O}$, Kasikara $\mathrm{H}$, et al. The effects of adding ischemic preconditioning during desflurane inhalation anesthesia or propofol total intravenous anesthesia on pneumoperitoneum-induced oxidative stress. Acta Chir Belg 2017;117:36-44.

12 Ates E, Yilmaz S, Ihtiyar E, et al. Preconditioning-Like amelioration of erythropoietin against laparoscopy-induced oxidative injury. Surg Endosc 2006;20:815-9.

13 Yilmaz S, Koken T, Tokyol C, et al. Can preconditioning reduce laparoscopy-induced tissue injury? Surg Endosc 2003;17:819-24.

14 Feng $\mathrm{Y}$, Jia R, Wu R, et al. [Effects of pneumoperitoneum preconditioning on endothelial progenitor cells and renal protective mechanism in rats]. Zhonghua Yi Xue Za Zhi 2015;95:1248-52.

15 Chan A-W, Tetzlaff JM, Altman DG, et al. SPIRIT 2013 statement: defining standard protocol items for clinical trials. Ann Intern Med 2013;158:200-7.

16 Haber G-P, Gill IS. Laparoscopic partial nephrectomy: contemporary technique and outcomes. Eur Urol 2006;49:660-5.

17 Chow SC, Wang H, Shao J. Sample Size Calculations in Clinical Research. 58. Second Edn. Chapman \& Hall/CRC Biostatistics Series, 2008.

18 Huang J, Chen Y, Dong B, et al. Effect of remote ischaemic preconditioning on renal protection in patients undergoing laparoscopic partial nephrectomy: a 'blinded' randomised controlled trial. BJU Int 2013;112:74-80.

19 Nesek-Adam V, Rasić Z, Vnuk D, et al. Ischemic preconditioning decreases laparoscopy induced oxidative stress in the liver. Coll Antropol 2010;34:571-6.

20 Bulbuloglu E, Yildiz H, Senoglu N, et al. Protective effects of zinc, pentoxifylline, and $\mathrm{N}$-acetylcysteine in an animal model of laparoscopy-induced ischemia/reperfusion injury of the small intestine. J Laparoendosc Adv Surg Tech A 2011;21:947-51.

21 Avraamidou A, Marinis A, Asonitis S, et al. The impact of ischemic preconditioning on hemodynamic, biochemical and inflammatory alterations induced by intra-abdominal hypertension: an experimental study in a porcine model. Langenbecks Arch Surg 2012;397:1333-41. 